\title{
Asociación Entre Variables Antropométricas y Actividad Física en Personal Administrativo Perteneciente a una Institución de Educación Superior en Colombia
}

\author{
RELATIONSHIP BETWEEN ANTHROPOMETRIY AND PHYSICAL ACTIVITY IN ADMINISTRATIVE STAFF BELONGING TO \\ A UNIVERSITY IN COLOMBIA
}

Armando Monterrosa Quintero', Antônio Renato Pereira Moro'

1. Centro Deportivo - CDS, Universidad Federal de Santa Catarina (Brasil).

\section{RESUMEN}

La actividad física está fuertemente ligada al desarrollo y mantenimiento de la composición corporal de las personas. El objetivo de este estudio fue analizar la asociación entre la actividad física y variables antropométricas de trabajadores de una universidad en Colombia. Participaron del estudio 84 hombres $(38,1 \pm 10$ años y $79,2 \pm 11 \mathrm{~kg})$ y 119 mujeres $(35,5 \pm 9,5$ años y $61,6 \pm 10,6 \mathrm{~kg})$. El nivel de actividad física fue evaluado utilizando los criterios del International Physical Activity Questionnaire. Fueron tomadas las medidas de peso, altura, IMC, porcentaje de grasa y masa magra. Fueron encontradas diferencias significativas en los niveles de actividad física bajo-alto con nivel medio en el peso $(p=0,021)$, y medio-alto con bajo en el porcentaje de grasa $(<0,001)$ y masa magra $(<0,001)$. Se encontró una asociación entre la actividad física con el IMC $(p=0,002)$. Se concluye que los trabajadores con nivel bajo de actividad física tienen mayor porcentaje de grasa y menos masa magra.

Quintero A, Pereira A, 2017. Asociación Entre Variables Antropométricas y Actividad Física en Personal Administrativo Perteneciente a una Institución de Educación Superior en Colombia. Cienc Trab. Sep-Dic; 19 [60]: 179-182).

Palabras claves: ANTROPOMETRÍA, ESFUERZO FÍSICO, COMPOSICIÓN CORPORAL, SALUD PÚBLICA, MOVILIDAD LABORAL.

\section{ABSTRACT}

Physical activity is strongly related to the development and maintenance of 1 body composition. The aim of this study was to analyze the association between physical activity and anthropometric variables of workers of a university in Colombia. 84 men (38.1 \pm 10 years and $79.2 \pm 11 \mathrm{~kg})$ and 119 women $(35.5 \pm 9.5$ years and $61.6 \pm$ $10,6 \mathrm{~kg}$ ) participated of the study. The level of physical activity was assessed using the criteria of the International Physical Activity Questionnaire. They were taken measures of weight, height, BMI, body fat percentage and lean mass. There were significant differences between low and high levels of physical activity in weight $(\mathrm{p}=0.021)$, between médium and high with low level for fat percentage $(<0.001)$ and lean mass $(<0.001)$ association was found between physical activity with BMI $(p=0.002)$. It is concluded that workers with low levels of physical activity have a higher percentage of fat and less lean mass.

Key words: ANTHROPOMETRY, PHYSICAL EXERTION, BODY COMPOSITION, PUBLIC HEALTH, CAREER MOBILITY.

\section{INTRODUCCIÓN}

La actividad física es definida como cualquier tipo de movimiento desarrollado por los músculos esqueléticos generando un gasto energético. ${ }^{1}$ Actualmente el sedentarismo (bajo nivel de actividad física) y los hábitos de vida no saludables tienen un alto porcentaje dentro de la población mundial. Un estudio realizado en

Correspondencia / Correspondence:

Armando Monterrosa Quintero

Universidad Federal de Santa Catarina

Trindade, Caja Postal 476 CEP 88040-900

Florianópolis, Santa Catarina, Brasil.

Tel.: 05548 99833-6029.

e-mail: adomonterrosa@gmail.com

Recibido: 02 de Junio de 2017 / Aceptado: 28 de Septiembre de 2017

Latinoamérica mostró que el 78\% de la población presenta inactividad física ${ }^{2}(30)$ y en otros 52 países se informó que el 85,73\% de la población sedentaria presentó una patología de tipo cardiaca con un valor de $80,72 \% .{ }^{3}$ En Colombia solamente un $21 \%$ de la población en edades de 18 a 64 años realiza actividad física en un periodo de tiempo de 150 minutos a la semana. ${ }^{4}$ A nivel de tiempo libre, el 34,3\% de la población cumple con las orientaciones de la OMS. Como medio de locomoción solo el 54,8\% realiza AF en sus horas no laborables. ${ }^{5}$ Este tipo de actividades diarias son menores en las mujeres que en hombres. ${ }^{4}$ A pesar de las diferencias en los géneros, el estudio desarrollado por la Encuesta Nacional de la Situación Nutricional 2010 (ENSIN 2010) ${ }^{6}$ mostró un nivel de interés medio-bajo en la población que practica actividad física en el ámbito educativo, teniendo en cuenta su estado nutricional, ocupación y región del país. ${ }^{6}$

Con relación a la locomoción teniendo como base la actividad física (caminar, correr, bicicleta, etc.), hay mayor prevalencia de 
este tipo de transporte en personas en edades comprendidas entre 50 y 64 años, con nivel socioeconómico y educativo de baja renta en distintas subregiones del país. ${ }^{7}$ Aunque algunos estudios mencionan prevalencias del $70 \%$ en las poblaciones sedentarias, existe un sinnúmero de procesos que afectan los apartados de estas investigaciones, mencionando la diversidad de métodos e instrumentos utilizados ${ }^{8}$, junto con promedios inconsistentes. ${ }^{9}$

Las diferentes metodologías de investigación en el campo del estudio corporal y la actividad física se basan en diferentes tipos de escalas dicotómicas (sedentario y activo), de niveles (bajo, medio y alto) y continuas (kilocalorías o METS). El estudio de la composición corporal especialmente en lo relacionado con el porcentaje de grasa, y el componente magro, se ha convertido en un tema de bastante interés entre los investigadores debido a la determinación de los valores de estas variables. ${ }^{10}$ A nivel mundial se evidencia un gran problema de salud pública por las enfermedades no transmisibles, que pueden ser en mayor parte por el continuo sedentarismo de las poblaciones y el aumento de alimentos pocos saludables. ${ }^{11}$

Algunos investigadores han determinado una fuerte correlación entre el aumento de grasa visceral y la diabetes tipo II en diferentes poblaciones. ${ }^{12}$ La grasa del pericardio está emergiendo como factor de alto riesgo en enfermedades de tipo coronario; la asociación inversa entre la actividad física y la grasa del pericardio fue más fuerte en adultos con sobrepeso y obesos que en los de peso normal. Niveles de actividad física evaluados objetivamente están relacionados con la grasa del pericardio, esto podría ser un mecanismo importante en la explicación del proceso de asociación entre la actividad física y la prevención de las enfermedades cardiovasculares. ${ }^{13}$ El comportamiento sedentario se asocia con resultados muy perjudiciales para la salud, que se atribuye a la falta de actividad física moderada y vigorosa. Esto se ha propuesto en el campo de la "fisiología sedentaria" que puede ser independiente y distinta con la fisiología del ejercicio. Los mecanismos propuestos por la fisiología sedentaria son examinados y difieren de la actividad física junto con el realce de la vinculación a la salud. ${ }^{14}$

La práctica de actividad física regular contribuye de formas positivas en la prevención, control de la obesidad y de las enfermedades crónico-degenerativas. ${ }^{15}$ Estudios sobre este tema han dado una gran contribución, significativa para nuestra sociedad; por medio de estos, se puede verificar en qué estado se encuentran los participantes de las investigaciones. De la misma forma se obtienen sugerencias para el cambio de estilos de vida y avances en las áreas relacionadas con la salud. ${ }^{16}$ En función de lo expuesto anteriormente, este estudio fue dirigido con el objetivo de analizar y comparar por medio de una investigación de tipo transversal los comportamientos de las variables que buscan evidenciar las características antropométricas de la composición corporal y de los niveles de actividad física de trabajadores de una universidad privada de la ciudad de Bucaramanga (Colombia).

\section{MÉTODOS}

El estudio aquí presentado es considerado de tipo correlacional basado en un diseño transversal debido a las situaciones que fueron realizadas las evaluaciones en una sola vez. La valoración fue realizada después del trabajo de condición física, con un tiempo determinado de 2 meses. Para la determinación de las variables se tuvo en cuenta el nivel asistencia al programa y las medidas antropométricas que fueron realizadas en los participantes del estudio.

\section{Población y muestra}

El procedimiento para determinar la muestra fue de tipo no probabilístico de tipo intencional, determinando un $n=203$ personas de los cuales 119 eran mujeres y 84 eran hombres, todos funcionarios de la Universidad participantes del Programa círculo educativo de promoción, prevención integral y social del ente educativo.

\section{Mediciones}

Para la evaluación antropométrica fue utilizada la balanza Swan (100 Gr), paquímetro, plicómetro, y cinta métrica, todas de marca Cescorf. Las medidas antropométricas fueron tomadas bajo protocolos de la International Society for advances of kinanthropometry. ${ }^{17}$ Para el porcentaje de grasa fue estimado midiendo los pliegues cutáneos de: Tríceps, bíceps, subescapular y cresta iliaca aplicando la ecuación de Durnin y Womersley. ${ }^{18}$ Los valores de la masa magra fueron determinados por medio de las medidas de: talla en metros, perímetros (brazo relajado, muslo relajado y pantorrilla) valores en centímetros; pliegues (tríceps, muslo anterior, pantorrilla) valores en milímetros; género y etnia utilizando la fórmula de Lee; Jamez. ${ }^{19}$

Para la determinación del Índice de Masa Corporal (IMC) se calculó con la fórmula: IMC = peso $(\mathrm{kg}) /$ talla $(\mathrm{m})^{2}$ y la clasificación (delgado < 18,5), normal 18,5-24,9). Sobrepeso (25 - 30) y obeso (más de 30)) se utilizaron los criterios propuesto por la Organización Mundial de la salud (OMS) en los que se utilizan los percentiles de IMC específicos para el género y la edad; estos valores posteriormente fueron transformados para el tratamiento estadístico. $^{15}$

La actividad física fue determinada por medio de niveles (bajo, medio y alto) y teniendo como factor la actividad física desarrollada en la semana, junto con los criterios del Cuestionario Internacional de Actividad Física (IPAQ) para la escala de dicha variable cualitativa. ${ }^{20}$

El objetivo del Programa círculo educativo de promoción, prevención integral y social del ente educativo, consiste en actividades encaminadas al desarrollo integral de los funcionarios de la universidad, abarcando áreas sociales, psicológicas, y de calidad de vida apoyadas por las directivas de la institución.

Los diferentes procedimientos, estructura y teorías éticas del estudio, fueron corroborados y apoyados por la oficina de Bienestar Universitario de la universidad; tras el apoyo de la institución, se realizó la publicidad del programa por los diferentes medios que posee el claustro universitario. Se informó a la comunidad que el estudio es de carácter voluntario y anónimo.

\section{Análisis estadístico}

Para la presentación de los datos fue realizada una estadística descriptiva (media \pm desviación estándar); la normalidad de los residuos de los datos fue verificada con el test KolgomorovSmirnov. Para verificar la asociación de los niveles de actividad física e IMC fue utilizado el test Chi-cuadrado; la determinación de las diferencias entre las variables antropométricas y los niveles de actividad física fue utilizado el test ANOVA one way. Las aplicaciones estadísticas se realizaron al nivel del 5\% de significancia. Para el análisis de datos se utilizó el paquete estadístico (SPSS para Windows v.17.0, SPSS, Inc., Chicago, IL. (USA). 


\section{RESULTADOS}

Los resultados obtenidos fueron divididos en cuatro partes. En primera medida presentamos los datos en la Tabla 1: la caracterización de la muestra, utilizando media y desviación estándar, de las variables edad, peso, altura, IMC, \% grasa y masa magra. En la Tabla 2 presentamos los valores de las diferencias entre las variables del estudio no encontrando diferencias significativas en las variables de edad, estatura e IMC comparados con los niveles de actividad física y fueron encontradas diferencias significativas en el peso entre los niveles bajo, alto, \% de grasa en niveles de medio,alto y masa magra entre niveles medio, alto.

La Tabla 3 describe los datos de la muestra de acuerdo a los niveles de actividad física. Se destaca el nivel IMC normal con un $63,4 \%$ comparado con el valor alto 36,6\%. El mayor número de personas se encuentra localizado en la posición normal con 114 personas, porcentaje de 56,2\% comparado con alto (89 personas) y porcentaje de 43,8\%. El valor del test de Chi cuadrado fue de $\mathrm{p}=0,002$, encontrándose un asociación entre las variables.

\section{DISCUSIÓN}

Destacamos en este estudio que más de la mitad de los participantes tienen un nivel bajo de actividad física. Este resultado incidió grandemente en los valores de las variables antropométricas (masa magra y porcentaje de gordura) del estudio de los funcionarios que pertenecen a la universidad, apoyándonos en lo propuesto por la OMS, donde ratifica la importancia del ejercicio de forma moderada en la prevención de las enfermedades no transmisibles y en la disminución de la tasa de mortandad en la población mundial, en especial en personas que laboran más de 4 horas en posiciones estáticas o de oficina. ${ }^{3}$

Resultados encontrados en un estudio en México mostró valores de las medias y desviación estándar de IMC en hombres $(28,9 \pm 5,2 \mathrm{~kg} /$ $\mathrm{m} 2)$; mujeres $(26,5 \pm 5,5 \mathrm{~kg} / \mathrm{m} 2) 8$, donde los datos obtenidos en

Tabla 1.

Características de la muestra (media \pm desviación estándar).

\begin{tabular}{lcc|} 
Variables & $\begin{array}{c}\text { Femenino } \\
\mathrm{n}=119\end{array}$ & $\begin{array}{c}\text { Masculino } \\
\mathrm{n}=84\end{array}$ \\
\hline Edad (años) & $35,5 \pm 9,5$ & $38,1 \pm 10,5$ \\
Peso $(\mathrm{kg})$ & $61,6 \pm 10,6$ & $79,2 \pm 11$ \\
Altura $(\mathrm{cm})$ & $161 \pm 6,1$ & $173 \pm 6,8$ \\
IMC $(\mathrm{kg} / \mathrm{m} 2)$ & $23,7 \pm 3,5^{*}$ & $26,3 \pm 3^{*}$ \\
Grasa $(\%)$ & $28,9 \pm 6,3$ & $22 \pm 5,8$ \\
Masa Magra $(\mathrm{kg})$ & $43,3 \pm 5,5$ & $61,4 \pm 7,1$
\end{tabular}

* Diferencias significativas ( $p \leq 0,001)$.

Tabla 2.

Diferencias entre variables teniendo en cuenta la actividad física.

\begin{tabular}{lccc} 
Variables & Bajo $(\mathrm{n}=131)$ & $\begin{array}{c}\text { Actividad Física } \\
\text { Medio }(\mathrm{n}=41)\end{array}$ & Alto $(\mathrm{n}=31)$ \\
\hline Edad (años) & $36,4 \pm 10,5$ & $38 \pm 9,6$ & $35,3 \pm 7,9$ \\
Altura (cm) & $164,7 \pm 8,4$ & $167,7 \pm 8,4$ & $168,4 \pm 10,2$ \\
Peso (kg) & $67,2 \pm 13,4^{*}$ & $74 \pm 14,3$ & $68,9 \pm 13,5^{*}$ \\
IMC (kg/m2) & $24,6 \pm 3,6$ & $26 \pm 3,5$ & $24 \pm 2,9$ \\
Grasa (\%) & $27,4 \pm 6,6$ & $24,3 \pm 6,2 \%$ & $22,4 \pm 7,8 \%$ \\
Masa magra (kg) & $48,5 \pm 9,7$ & $56 \pm 11,3 \#$ & $53,5 \pm 12,2 \#$
\end{tabular}

$\mathrm{P}<0,05$ * Diferente del nivel medio, \# diferente del nivel bajo, \% Diferente del nivel bajo.
Tabla 3.

Asociación cualitativa de variables.

\begin{tabular}{|c|c|c|c|c|c|}
\hline \multicolumn{3}{|c|}{$\begin{array}{l}\text { Los valores fueron } \\
\text { determinados según la OMS }\end{array}$} & Normal & $\begin{array}{l}\text { IMC } \\
\text { Alto }\end{array}$ & Total \\
\hline \multirow{4}{*}{$\begin{array}{l}\text { Categorias } \\
\text { actividad } \\
\text { fisica }\end{array}$} & Bajo & $\begin{array}{l}\text { Personas } \\
\text { porcentaje }\end{array}$ & $\begin{array}{l}83 \\
63,4 \%\end{array}$ & $\begin{array}{c}48 \\
36,6 \%\end{array}$ & $\begin{array}{c}131 \\
100,0 \%\end{array}$ \\
\hline & Medio & $\begin{array}{l}\text { Personas } \\
\text { porcentaje }\end{array}$ & $\begin{array}{l}13 \\
31,7 \%\end{array}$ & $\begin{array}{c}28 \\
68,3 \%\end{array}$ & $\begin{array}{c}41 \\
100,0 \%\end{array}$ \\
\hline & Alto & $\begin{array}{l}\text { Personas } \\
\text { porcentaje }\end{array}$ & $\begin{array}{l}18 \\
58,1 \%\end{array}$ & $\begin{array}{c}13 \\
41,9 \%\end{array}$ & $\begin{array}{c}31 \\
100,0 \%\end{array}$ \\
\hline & Total & $\begin{array}{l}\text { Personas } \\
\text { porcentaje }\end{array}$ & $\begin{array}{l}114 \\
56,2 \%\end{array}$ & $\begin{array}{c}89 \\
43,8 \%\end{array}$ & $\begin{array}{c}203 \\
100,0 \%\end{array}$ \\
\hline
\end{tabular}

hombres $\left(26,3 \pm 3 \mathrm{~kg} / \mathrm{m}^{2}\right)$, mujeres $\left(23,7 \pm 3,5 \mathrm{~kg} / \mathrm{m}^{2}\right)$ muestran una tendencia menor en nuestra población. Comparando la función laboral que desempeñan las personas y los niveles de actividad física, en este caso México (profesores de Educación física) y Colombia (personal administrativo), logramos definir que el trabajo de actividad física desarrollado ha sido satisfactorio en comparación con otros realizados en la misma área.

El valor de la obesidad y sobrepeso mencionado en esta investigación fue de $43,8 \%$ y otras realizadas en Colombia reportaron una prevalencia de obesidad y sobrepeso: $45,8 \%{ }^{21}, 42 \%(6)^{22}, 43,3 \%(9)^{23}$,- en Chile 47,7\% (20) ${ }^{24}$ y México 37,8\% (10). ${ }^{8}$ En la actualidad, el sobrepeso y la obesidad cada día ganan más terreno dentro la población mundial y se consideran como nuevas epidemias que disminuyen la calidad de vida de las personas (4). ${ }^{25}$ Un estudio al respecto realizado por Framignam informa sobre la obesidad como un factor muy nocivo para la salud pública (18). ${ }^{26}$ En nuestro estudio las personas que tuvieron mayor IMC no eran las que tenían menor nivel de AF -cuando confrontamos la actividad física con las demás variables, podemos identificar que la actividad física comparada con el IMC no presentan diferencias significativas-, pero sí se presenta una asociación $(\mathrm{p}=0,002)$. Teniendo en cuenta lo anterior, deducimos que el proyecto institucional es considerado de vital importancia para el desarrollo integral de los funcionarios dentro de lo establecido por la OMS y la organización mundial de trabajo (21). ${ }^{19}$ Apoyándonos en la literatura encontrada para este artículo, podemos decir que el tiempo no fue considerado suficiente, analizando que la cantidad de tiempo de los funcionarios es ocupado por jornada laboral (8 horas) y las demás dedicadas a su vida personal y actividades familiares. Procedimientos encaminados al mejoramiento de la calidad de vida en los funcionarios de empresas han demostrado eficiencia en diferentes factores sociales. En países de norteamérica los valores de muertes por las enfermedades de tipo no trasmisibles han disminuido en proporción: las campañas de prevención y apropiación de hábitos saludables y en especial tener un modo de vida activo se consideran importantes para estos objetivos (15). ${ }^{27}$

La actividad física desarrollada de forma constante previene los factores que pueden incidir en las patologías de tipo cardiaca, accidentes cerebro-vasculares, descalcificación, estado de ánimo, enfermedades no transmisibles e inclusive riesgo de adquirir cáncer (22). ${ }^{28}$ Las evidencias de la bondad de practicar ejercicios físicos determinan la importancia de la prevención y promoción de la salubridad pública.

En estudios realizados se ha comprobado que las personas que realizan algún tipo de actividad física tienen un periodo de vida mayor que las que no se ejercitan; en los adultos mayores quienes realizan actividad física de forma moderada se ha identificado una mejor independencia a la hora de realizar sus actividades diarias con un nivel de seguridad mayor debido al riesgo de presentar algún tipo de caída (28). ${ }^{29}$ 
Los proyectos encaminados a los hábitos de vida saludable se han convertido hoy en día en procedimientos indispensables en la manutención y mejoramiento de la salud del trabajador. Al motivar a la comunidad universitaria permitirá aumentar los niveles de funcionalidad e integración que incidirá grandemente en los aspectos sociales, físicos y sicológicos.

Una de las limitaciones en esta investigación es la comparación de datos entre el proyecto inicial y otro secundario; también la disponibilidad de horarios para la toma de datos, la motivación a las campañas de bienestar universitario apoyadas por los diferentes profesionales participantes de esta investigación.

Teniendo en cuenta la información aquí presentada, es de vital importancia mantener el programa de actividad física dentro de la institución como mecanismo de mejora, prevención y control de alteraciones, especialmente dirigidas a la comunidad universitaria. Por lo tanto, el objetivo del programa es contribuir en la disminución de las variables estudiadas, nocivas para la salud en los funcionarios de la universidad.

\section{CONCLUSIONES}

Los resultados del estudio indican una asociación entre la actividad física y el IMC entre los niveles alto-bajo con medio en el peso, medio-alto con bajo en el porcentaje de grasa y masa magra de las personas que laboran en la institución. A pesar de que algunos participantes presentaron un nivel bajo de Actividad Física, se concluyó que los trabajadores tienen mayor porcentaje de grasa y menos masa magra.

Podemos decir que es importante mantener el programa de calidad de vida en la universidad. En comparaciones realizadas con otros estudios, apoyamos los diferentes conceptos sobre el nivel de mortalidad de la población mundial debido a la inactividad física. Se observó que los datos presentados por los entes que regulan la salud en Colombia, no están alejados de los datos aquí presentados y que es de vital importancia la continuidad del proyecto como ejemplo ante las demás comunidades universitarias del área Metropolitana de Bucaramanga.

\section{REFERENCIAS}

1. Shephard R. Exercise as cardiovascular therapy. Circulation 1999; 99(7):963972.

2. Yusuf S. Effect of potentially modifiable risk factors associated with myocardial infarction in 52 countries (the INTERHEART study): case-control study. Lancet. 2004; 364(9438):937-952.

3. Vidarte A. Actividad física: estrategia de promoción de la salud. Hacia Promoc Salud. 2011; 16(1):202-218.

4. Colombia. Ministerio de Salud-Observatorio Nacional de Salud. Carga de enfermedad por enfermedades crónicas no transmisibles y discapacidad en Colombia. Bogotá: Ministerio de Salud; 2015. (Informe Técnico ONS, 5).

5. Serón P. Nivel de actividad física medida a través del cuestionario internacional de actividad física en población chilena. Rev Med Chile. 2010; 138(10):1233-1239.

6. Colombia. Ministerio de Protección Social. Encuesta Nacional de la Situación Nutricional en Colombia-ENSIN. Bogotá: ICBF; 2011.

7. Brown W. Comparison of surveys used to measure physical activity. Aust $\mathrm{N}$ Z J Public Health. . 2004; 28: (128-134).

8. Hall J, Ochoa P, Alarcón E. Actividad física, estado nutricional y obesidad abdominal en profesores del área de la cultura física. Rev Int Med Cienc Act Fis Deporte. 2012; (46):209-220.

9. Heyward V. Avaliação da composição corporal aplicada. São Paulo: Manole; 2000.

10. Henson J, Edwardson CL, Morgan B, Horsfield MA, Bodicoat DH, Biddle SJ, Gorely T, Nimmo MA, McCann GP, Khunti K, Davies MJ, Yates T. Associations of Sedentary Time with Fat Distribution in a High-Risk Population. Med Sci Sports Exerc. 2015; 47(8):1727-1734.

11. Farias E. Antropometría, composição corporal e atividade física de escolares. Rev Bras Cineantropom Desempenho Hum. 2005; 7(1): 21.

12. Hamer M, Venuraju SM, Urbanova L, Lahiri A, Steptoe A. Physical activity, sedentary time, and pericardial fat in healthy older adults. Obesity. 2012; 20(10):2113-2117.

13. Stephen M. Physiological and health implications of a sedentary lifestyle. Appl Physiol Nutr Metab. 2010; 35(6):725-740.

14. Buonani C. Prática de atividade física e composição corporal em mulheres na menopausa. Rev Brasil Ginecol Obstet. 2013; 35(4):153-158.

15. Gallagher D, Heymsfield SB, Heo M, Jebb SA, Murgatroyd PR, Sakamoto Y.
Healthy percentage body fat ranges: an approach for developing guidelines based on body mass index. Am J Clin Nutr. 2000; 72(3):694-701.

16. Beltrán C. Actividad física y sedentarismo en adolescentes de la Comunidad Valenciana. Rev Int Med Cienc Act Fis Deporte.2012; 12(45):122-137.

17. Carter JE. Estandarización en antropometría. Glasgow: ISAK; 2015.

18. Durnin J, Womersley J. Body fat assessed from total body density and its estimation from skinfold thickness: measurements on 481 men and women aged from 16 to 72 years. Br J Nutr. 1974; 32(1):77-97.

19. Lee KN, Kritchevsky D, Pariza MW. Conjugated linoleic acid and atherosclerosis in rabbits. Atherosclerosis. 1994; 108(1):19-25.

20. Matsudo S. Questionário Internacional de Atividade Física (I PAQ). Rev Bras ativ fis saúde. $2001 ; 6(2): 5-18$.

21. Jesús E. Factores de riesgo para enfermedad cardiovascular en trabajadores de una Institución prestadora de servicios de salud, Colombia. Rev Salud Pública (Bogotá). 2007; 9(1):64-75.

22. Díaz M, Córdoba D. Identificación de factores de riesgo de enfermedad cardiovascular presentes en los pacientes que ingresan al Hospital San Ignacio. Lect Nutr. 2003; 10(4):51-58.

23. Gómez L, Samper B, Cabrera G, Espinosa G, Mateus J. Factores de riesgo cardiovascular en la localidad de Santa Fe de la ciudad de Bogotá. Resultados obtenidos en el área demostrativa Carmen. Investig Segur Soc Salud. 2004; 6:11-24.

24. Landers D. Physical Activity, fitness and anxiety. En: Bouchard C, Shephard $R$, Stephens T, editores. Physical activity, fitness and Health. Illinois: Human Kinetics; 1994. p. 868-882.

25. Bustos P. Factores de riesgo de enfermedad cardiovascular en adultos jóvenes. Rev Méd Chile. 2003; (131):973-978.

26. James P. Obesity: the worldwide epidemic. Clin Dermatol. 2004; (22):276280.

27. Hubert H. Obesity as an independent risk factor for cardiovascular disease: a 26-year follow-up of participants in the Framingham Heart Study. Circulation. 1983; 67(5):968-977.

28. Martínez E. La actividad física en el ámbito de la Salud Pública. Rev Fac Nac Salud Pública. 1998; 15(2):140-153.

29. Swain D. Comparison of cardioprotective benefits of vigorous versus moderate intensity aerobic exercise. Am J Cardiol. 2006: 97(1):141-147. 